\title{
An Indoor Test Methodology for Solar-Powered Wireless Sensor Networks
}

\author{
WILSON M. TAN and PAUL SULLIVAN, University of Warwick \\ HAMISH WATSON and JOANNA SLOTA-NEWSON, Polysolar Ltd \\ STEPHEN A. JARVIS, University of Warwick
}

\begin{abstract}
Repeatable and accurate tests are important when designing hardware and algorithms for solar-powered wireless sensor networks (WSNs). Since no two days are exactly alike with regard to energy harvesting, tests must be carried out indoors. Solar simulators are traditionally used in replicating the effects of sunlight indoors; however, solar simulators are expensive, have lighting elements that have short lifetimes, and are usually not designed to carry out the types of tests that hardware and algorithm designers require. As a result, hardware and algorithm designers use tests that are inaccurate and not repeatable (both for others and also for the designers themselves). In this article, we propose an indoor test methodology that does not rely on solar simulators. The test methodology has its basis in astronomy and photovoltaic cell design. We present a generic design for a test apparatus that can be used in carrying out the test methodology. We also present a specific design that we use in implementing an actual test apparatus. We test the efficacy of our test apparatus and, to demonstrate the usefulness of the test methodology, perform experiments akin to those required in projects involving solar-powered WSNs. Results of the said tests and experiments demonstrate that the test methodology is an invaluable tool for hardware and algorithm designers working with solar-powered WSNs.
\end{abstract}

Categories and Subject Descriptors: C.4 [Computer Systems Organization]: Performance of Systems; C.2.4 [Computer Systems Organization]: Computer Communication Networks

General Terms: Experimentation, Measurement, Performance, Theory

Additional Key Words and Phrases: Energy harvesting, astronomical models, solar simulators, lifetime, power management, energy neutrality

\section{ACM Reference Format:}

Wilson M. Tan, Paul Sullivan, Hamish Watson, Joanna Slota-Newson, and Stephen A. Jarvis. 2017. An indoor test methodology for solar-powered wireless sensor networks. ACM Trans. Embed. Comput. Syst. 16, 3, Article 82 (March 2017), 25 pages.

DOI: http://dx.doi.org/10.1145/2994604

This work is funded in part by the UK Technology Strategy Board (TSB) Emerging Technologies Programme, Project 131187/26835-183208, Keeping the Noise Down: OPV-Based Energy Harvesting Sensors for Urban Noise Pollution. W. M. Tan is supported in part by the Republic of the Philippines' Engineering Research and Development for Technology (ERDT) Program.

Authors' addresses: W. M. Tan, now at the Department of Computer Science, University of the PhilippinesDiliman, Quezon City, Metro Manila, Philippines; email: wmtan@dcs.upd.edu.ph; P. Sullivan, Department of Chemistry, University of Warwick, Coventry, England, United Kingdom; H. Watson and J. Slota-Newson, Polysolar Ltd, Hauser Forum, Charles Babbage Road, Cambridge, England, United Kingdom; S. A. Jarvis, Department of Computer Science, University of Warwick, Coventry, England, United Kingdom; email: s.a.jarvis@warwick.ac.uk.

Permission to make digital or hard copies of all or part of this work for personal or classroom use is granted without fee provided that copies are not made or distributed for profit or commercial advantage and that copies bear this notice and the full citation on the first page. Copyrights for components of this work owned by others than the author(s) must be honored. Abstracting with credit is permitted. To copy otherwise, or republish, to post on servers or to redistribute to lists, requires prior specific permission and/or a fee. Request permissions from permissions@acm.org.

2017 Copyright is held by the owner/author(s). Publication rights licensed to ACM.

ACM 1539-9087/2017/03-ART82 $\$ 15.00$

DOI: http://dx.doi.org/10.1145/2994604 


\section{INTRODUCTION AND MOTIVATION}

A significant challenge in power management algorithm and hardware design for solarpowered wireless sensor networks (WSNs) is the repeatability of experiments. Sunlight patterns are highly variable, seasonally varying in intensity and length, and strongly affected by local weather phenomena. Most power management algorithms for solarpowered WSNs are therefore tested via simulations (for example, see Chetto and Zhang [2010] and Moser et al. [2006]) or through simple tabletop experiments that involve lamps being turned on or off (for example, Lee et al. [2010]). Both approaches overlook a great deal of detail that is central for WSN operation. The accuracy and level of detail of the simulations are highly variable; they also frequently neglect hardware-specific features, such as non-idealities in the energy storage device characteristics. Most tabletop experiments (such as that documented in Lee et al. [2010]) utilize actual hardware but still omit the effects of daylight patterns: As a result, the nodes are possibly exposed to unrealistic conditions that they will never encounter in actual deployments (the ease with which this can be done will be demonstrated later in the article).

Repeatable experiments involving sunlight are also frequently needed in the field of photovoltaic (PV) cell (also called solar cell) design. In this domain, this problem is approached with the help of solar simulators (for example, Oriel Instruments [2012]). Solar simulators generate light that approximates the intensity and the spectral content of natural sunlight. Most solar simulators, however, are designed for single-intensity exposure experiments: They subject the solar cells to a constant specific light intensity. They are not designed to automatically replicate the changing intensity of sunlight throughout the day. The intensity and duration of light to which a solar-powered WSN node is exposed are of primary importance to hardware and algorithm designers: For a given hardware (component sizing) and software (algorithm parameters) setup, they determine the system's performance and survivability-it is therefore important for WSN nodes to be tested with irradiation patterns that mirror real-world conditions. Moreover, solar simulators are expensive and bulky devices, with lighting elements that have very limited lifetimes.

In this work, we present a test methodology that enables repeatable indoor testing of solar-powered WSN nodes without the use of a solar simulator in the test itself. The test methodology induces the solar cell or solar panel to generate a level of power that it will exhibit under outdoor conditions at a specific time and place. The methodology has its basis on insights from PV cell design and astronomy. We first present the test methodology in an apparatus-agnostic manner, specifying the general principles of the test apparatus design. To prove its practicality, however, we present our own specific design and implementation of the test apparatus. We present two variant designs for the apparatus. The first variant can be used in testing standalone wireless sensor network nodes. The second variant is a distributed version of the first and can be used as a basis for testbeds designed to test wireless sensor networks. The performance and accuracy of the test methodology and our test apparatus are verified via a series of experiments. Moreover, we also carry out a series of demonstration experiments, the likes of which will be useful in studies aiming for outdoor deployment of solar-powered WSNs. It must be noted that while this article focuses on WSNs and WSN nodes, the test methodology is also readily applicable to any solar-powered embedded system, even those that are not designed to be networked.

In summary, we make two primary contributions in this article. First, the indoor test methodology, and the design for a generic test apparatus that can be used in its implementation, and, second, the design of an actually implemented apparatus. We present two variants of the apparatus: centralized and distributed. We also present the results of two sets of experiments: the first tests the performance of the test apparatus, 
while the second is composed of experiments that demonstrate how the methodology can be used in determining hardware and software parameters for a WSN node.

The remainder of this article is structured as follows. The test methodology is presented in the following section (Section 2). The design of a generic test apparatus on which the test methodology can be implemented is presented in Section 3. Section 4 discusses our specific design and implementation of the test apparatus. We test the performance of our test apparatus and present the results of the said tests in Section 5. The results of two experiments that demonstrate the utility and possible applications of the test methodology are presented in Section 6; limitations are discussed in Section 7. Related work is discussed in Section 8. Section 9 presents possible future extensions of the research and concludes the article.

\section{TEST METHODOLOGY}

Before outlining the general principles of the test methodology, we need to understand the relationship between a surface's irradiance, the surface's location, and the current date and time; this will be discussed in Section 2.1. An understanding of a solar cell's "state" and how such a state can be determined is also required and this will be discussed in Section 2.2. The general principles built from these two examinations are presented in Section 2.3.

\subsection{Astronomical Model of Irradiance}

Irradiance (unit $\frac{W}{m^{2}}$ ) is the total power from a radiant source falling onto a unit area. A detailed discussion of how irradiance due to the sun is computed at a given location, time of day, and time of year is given in Messenger and Ventre [2010] and Markvart [2000]. In this section, we give a necessarily brief summary of the procedure.

The power density in sunlight that reaches the Earth from the sun is $1,367 \frac{\mathrm{W}}{\mathrm{m}^{2}}$. However, some of this is absorbed in the atmosphere, so the power density that reaches the surface of the Earth is less. The amount of sunlight absorbed or scattered depends on the length of path through which sunlight has to travel to get to the surface. The path length is generally compared to a path directly vertical to sea level. This path length is designated as 1 atmosphere or AM 1. At AM 1, after absorption is accounted for, the power density is reduced from $1,367 \frac{\mathrm{W}}{\mathrm{m}^{2}}$ to $1,000 \frac{\mathrm{W}}{\mathrm{m}^{2}}$. Assuming that the absorption constant depends only on the air mass, and taking $I$ as the irradiance due to the sun, we have Equation (1) as follows:

$$
I=1,367(0.7)^{A M} .
$$

However, Meinel and Meinel [1976] proposed that a better fit to observed data is obtained through Equation (2) as follows:

$$
I=1,367(0.7)^{A M^{0.678}} .
$$

$\mathrm{AM}=1$ when the rays from the sun are directly over the surface in consideration. The AM for any ray source direction is given by Equation (3) (all angles stated in this section are in degrees):

$$
A M=\frac{1}{\cos \theta_{z}},
$$

where $\theta_{z}$ is the zenith angle, which is the complement to $90^{\circ}$ of the elevation $\alpha$. The elevation is the angle between the sun's direction and the horizon.

$$
\theta_{z}=90-\alpha
$$




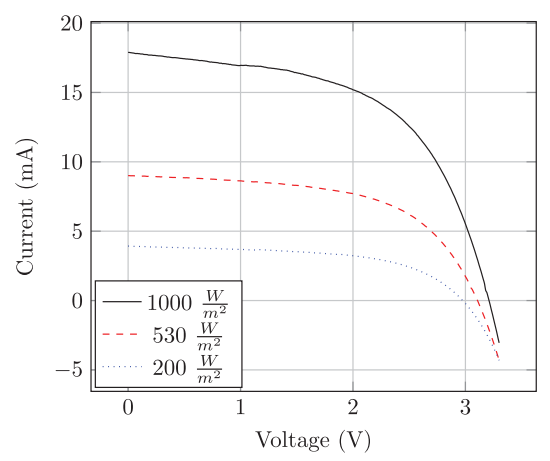

Fig. 1. IV curves of a solar panel.

The elevation, or $\alpha$, can be derived from Equation (5) as follows:

$$
\alpha=\sin ^{-1}(\sin \delta \sin \theta+\cos \delta \cos \theta \cos \omega) .
$$

$\theta$ is the latitude of the surface. $\delta$ is the solar declination, or the angle between the line joining the centres of the Earth and the sun, and the equatorial plane. This can be derived from Equation (6) as follows:

$$
\delta=23.45 \times \sin \left(360 \times \frac{n-180}{365}\right),
$$

where $n$ is the day in the year ( $n=1$ on 1 January). $\omega$ is the angle difference between noon and the desired time of day in terms of a $360^{\circ}$ rotation in $24 \mathrm{~h}$ :

$$
\omega=\frac{12-T}{24} \times 360=15(12-T),
$$

where $T$ is the time of day with respect to solar midnight on a $24 \mathrm{~h}$ clock.

In summary, given the location of a surface, and the current date and time of day of interest, the irradiance due to the sun can be computed.

\subsection{Solar Cell State}

Each solar cell has an associated set of current-voltage (IV) curves (Figure 1). The currently relevant IV curve is dependent on the irradiance of the solar cell, while the specific point on the curve (the solar cell's current operating point) is dependent on the electrical load imposed on the solar cell. The currently relevant IV curve for the solar cell can be determined by measuring the cell's short circuit current $\left(I_{S C}\right)$, which is unique for each level of irradiance. The relationship between the irradiance and the $I_{S C}$ is highly linear [Messenger and Ventre 2010]: For instance, if the $I_{S C}$ at $1000 \frac{\mathrm{W}}{\mathrm{m}^{2}}$ is $18.9 \mathrm{~mA}$, the $I_{S C}$ at $500 \frac{\mathrm{W}}{\mathrm{m}^{2}}$ will be approximately $9.45 \mathrm{~mA}$. We can consider the currently relevant IV curve as the solar cell's state. It must be noted that how a solar cell is induced to reach a certain state is not relevant here. Two similar cells, exposed to different light sources (different spectral content and intensities) can be said to be statewise the same, as long as they have the same currently relevant IV curve. This is the key concept that enables us to replace the lighting element in traditional solar simulators with a relatively low-power, cheaper, and more easily controlled alternative. 


\subsection{General Principles}

We are now in a position to state the general principles of the test methodology

(1) Irradiance for a surface at a certain place, date, and time can be computed using the set of equations presented in Section 2.1. Irradiance sequences representing days (or parts of days) can be generated by iteratively solving the set of equations. It must be noted that, in reality, the brightness of sunlight is continuously changing during the day; the irradiance therefore can actually be better modeled as a continuous function rather than a discrete sequence. However, we will be implementing the test methodology using discrete-time systems, so some discretization is necessary. We define the time interval between changes in irradiance values as the temporal resolution of a sequence.

(2) The currently relevant IV curve (hence, the irradiance of the solar cell) can be determined by measuring the solar cell's $I_{S C}$. By taking advantage of the linear relationship between the $I_{S C}$ and the irradiance level, an irradiance sequence can therefore be converted to an $I_{S C}$ sequence.

(3) The simulation of a day (or part of it) can then be carried out by inducing the solar cell to sequentially produce the $I_{S C}$ values specified in the $I_{S C}$ sequence.

A single solar cell usually cannot produce enough voltage or current to power an application. As such, what are used as energy harvesting devices are solar panels, which are similar solar cells placed in a series and/or parallel configuration to increase the output voltage and/or current. Like solar cells, solar panels also have IV curves, and their characteristics are derived from that of their component solar cells. Since most devices (including those used in this work) use solar panels and not simply solar cells, we refer to the energy harvesting device in subsequent sections as solar panels.

\section{GENERIC TEST APPARATUS DESIGN}

A generic test apparatus that can carry out the test methodology will need three components.

(1) Light source. The light source's output does not need to have the same spectral content as sunlight. However, it must coincide (even if not completely) with the frequencies to which the solar panel is sensitive. The light source must be able to induce the solar panel to enter states corresponding to different irradiance levels, including the irradiance level of $1000 \frac{\mathrm{W}}{\mathrm{m}^{2}}$, also known as 1 sun (the maximum irradiance due to the sun possible at the surface of the Earth).

(2) Current measurement mechanism. A generic test apparatus must be able to measure the solar panel's $I_{S C}$ or a suitable proxy to it. There are two challenges associated with measuring the $I_{S C}$ : where and how it must be measured.

The test methodology utilizes $I_{S C}$ for determining the current state of the solar panel. However, measuring $I_{S C}$ requires that the solar panel be in a short circuit, which is not the case when it is in a useful configuration (i.e., in a circuit). The problem can be solved by utilizing proxy $I_{S C}$ measurements. Two measurements can serve as proxies for the $I_{S C}$ : the $I_{S C}$ of a pilot panel and the output of the panel itself.

We define a pilot panel as a solar panel that is of the same type as the solar panel found in the device-under-test (DUT) and co-located with it. Assuming that the two panels have the same characteristics, and are under the same lighting conditions, they will be in the same state (since the currently relevant IV curve is defined only by the irradiance of the panel's surface). The $I_{S C}$ of the pilot panel can then be measured and used in determining the state of both panels. The schematic of 


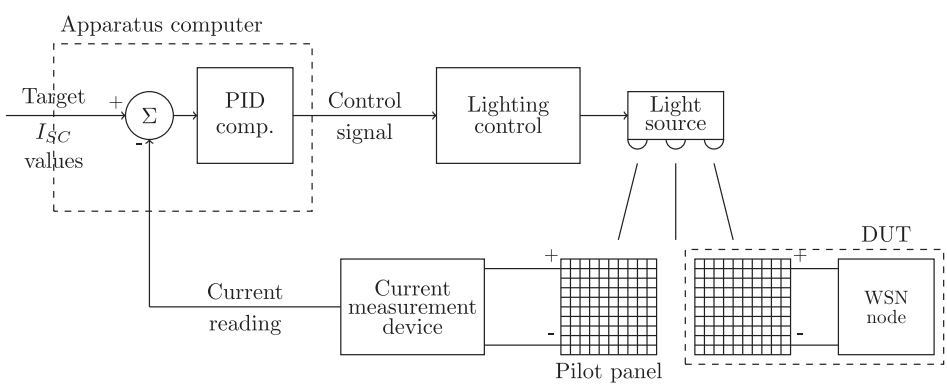

(a) Using the pilot panel $I_{S C}$ as proxy

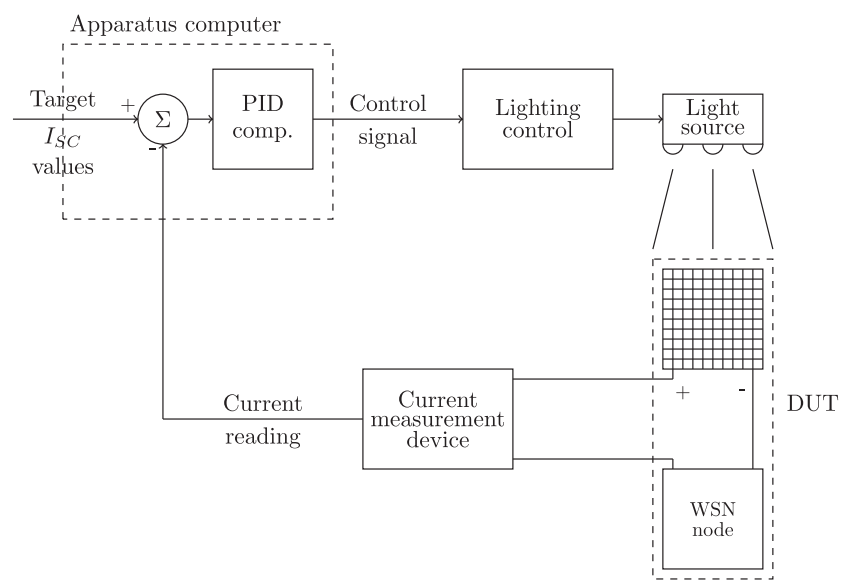

(b) Using the solar panel current as proxy

Fig. 2. Generic test apparatus design schematic.

a generic test apparatus that uses the pilot panel $I_{S C}$ as proxy for the DUT solar panel $I_{S C}$ is shown in Figure 2(a).

The actual current of the solar panel can also be used as a proxy to a limited extent. This is due to the current value remaining constant (the same as the $I_{S C}$ ) for the most part of an IV curve (Figure 1, left-side portion). However, depending on the electrical load, this may not always be the case (see right-most part of Figure 1). The schematic of a generic test apparatus that uses the actual solar panel current as proxy for the $I_{S C}$ is shown in Figure 2(b).

An additional challenge is the process of measuring the current itself. Accurate current measurement is a complex process. Most microcontrollers have analog-todigital converters that measure voltage but not current. This limits the prospect of the operation being done in distributed systems with low complexity. The current measurement process can be converted to a voltage measurement process with the use of shunt resistors. Shunt resistors, however, introduce losses, which may be unacceptable when the current is small to begin with. The losses due to a shunt resistor can be minimized by using smaller shunt resistor values. This is made feasible using current sense amplifiers such as the LT6105 [Linear Technology 2013].

(3) Feedback mechanism. A generic test apparatus must have a feedback mechanism that will adjust the lighting element's output in response to the difference between the actual-measured $I_{S C}$ and the target $I_{S C}$. A feedback loop can be 
implemented using a Proportional-Integrative-Derivative (PID) controller [Astrom and Hagglund 1995]. The P, I, and D constants have to be carefully derivedimproperly set constants can lead to an unstable system, with the output variable (the $I_{S C}$ in this case) never converging to a specific value. The performance of the PID controller will also depend on the accuracy of the current measurements.

The design presented above is generic: It is a design that in theory will enable any type of light source to be used in the apparatus (as long as the spectral content requirements stated above are satisfied). An actual implementation does not need to perfectly conform with the generic design; what is important is that the apparatus is able to induce the solar panel to produce the $I_{S C}$ values contained in the $I_{S C}$ sequence.

Our own implementation of the test apparatus features neither a current measurement mechanism nor a feedback loop. We justify the omission with our choice of a light source that is sufficiently stable. The details of our test apparatus design, our definition of stability, and its demonstration vis-à-vis our design, are presented next.

\section{TEST APPARATUS IMPLEMENTATION}

We construct two variants of the test apparatus: one for testing WSN nodes, which we call the centralized test apparatus (see Section 4.1), and another that can be used for testing networks of WSN nodes, which we call the distributed test apparatus (see Section 4.2).

\subsection{Centralized Test System}

The lighting element in our implemented test apparatus (both centralized and distributed) is the Luxeon K 8-Light Emitting Diode (LED) array (LXK8-PW40-0008 [Lumileds 2014]). The light intensity of the Luxeon $\mathrm{K}$ is current controlled by the buck regulator-controlled current source LM3406HV [Texas Instruments 2013]. The output of the LM3406HV is then controlled via pulse width modulation (PWM). In the centralized version of our apparatus, the PWM signal is generated by an Arduino Uno [Arduino 2013] (interfaced to a Personal Computer (PC) via Universal Serial Bus (USB) cable). In the distributed version, the PWM signal is generated by a TelosB WSN node [Polastre et al. 2005]. For the centralized test apparatus, the Luxeon $\mathrm{K}$ is interfaced with a heatsink and mounted on a platform that enables the adjustment of the vertical distance between the LED array and the DUT.

Our choice of components (both for the centralized and the distributed version of our apparatus) is driven by two factors: (1) the availability of components and (2) the size of the DUT's solar panel (this is particularly important in choosing the light source and designing the adjustable platform). As stated in Section 3, a test apparatus can be built from several different types of components, but it must be ensured that the light source can drive the $I_{S C}$ of the DUT's solar panel to its level at $1 \mathrm{sun}$. We ascertained that this requirement is met with the use of a sourcemeter (this will be demonstrated later; the result can be seen in Figure 5(b)).

Figure 3 shows the schematic of our centralized test apparatus; the actual test apparatus is shown in Figure 4.

The feedback loop (and its current measurement input) is necessary for dynamically adjusting the lighting element in response to the difference between the measured $I_{S C}$ and the target $I_{S C}$. This is particularly important for light sources with resulting $I_{S C}$ values that tend to drift even with a constant light source setting. Some bulbs, for example, glow brighter as their filaments heat up, even if the supply voltage remains constant. A feedback loop is not necessary in situations where the resulting $I_{S C}$ for a given lighting element setting is distinct and unchanging. We call the tendency of a light source to have a distinct and unchanging $I_{S C}$ at each setting its stability. We note 


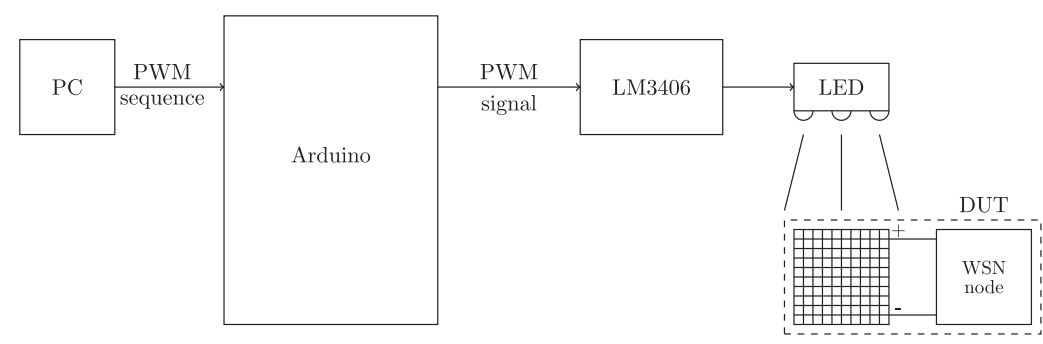

Fig. 3. Design schematic of the implemented centralized test apparatus.

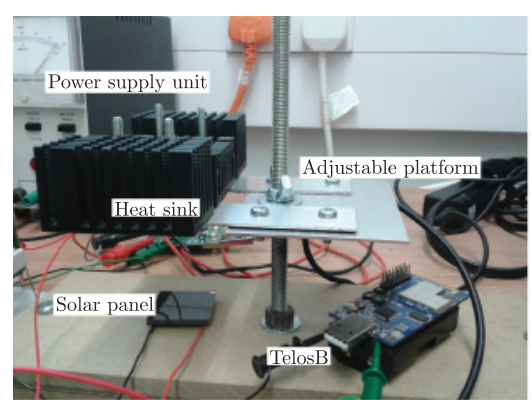

(a) Lighting element (under the heatsink) and vertically-adjustable stand

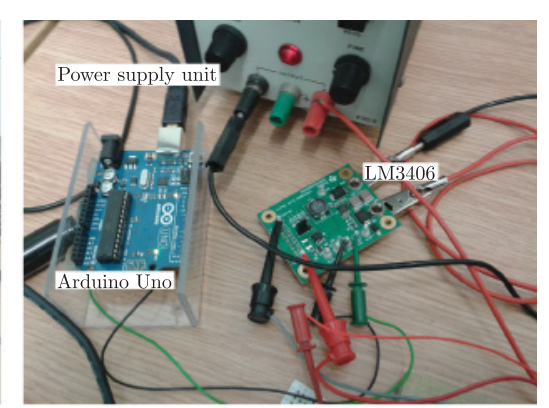

(b) Arduino and LM3406

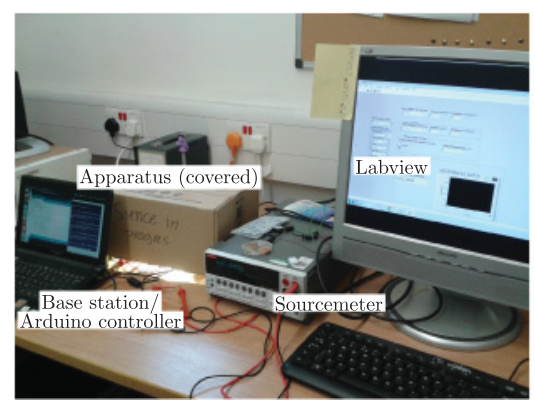

(c) Test apparatus in operation, with base station and sourcemeter

Fig. 4. Centralized test apparatus implementation.

that by "light source" we mean the lighting element along with the mechanism that controls its settings. In our specific setup, the light source is collectively composed of the LED array, the LM3406HV, and the Arduino.

We quantitatively define stability using two metrics. First, we measure the variance of the solar panel $I_{S C}$ observed at a given setting. The variance provides a measure of how much the $I_{S C}$ fluctuates. The lower the variance, the more stable the light source is at that setting. A perfectly stable light source has a variance of 0 for all sets of $I_{S C}$ readings. Second, we measure the tendency of the $I_{S C}$ to rise or fall over time using the trendline slope. Using linear regression, we generate a trendline for the values observed at a given setting and take note of its slope. This is done for all settings tested. The closer to 0 the trendline slope is, the more stable the light source is at that setting. A perfectly stable light source has a trendline slope of 0 for all sets of $I_{S C}$ readings. 


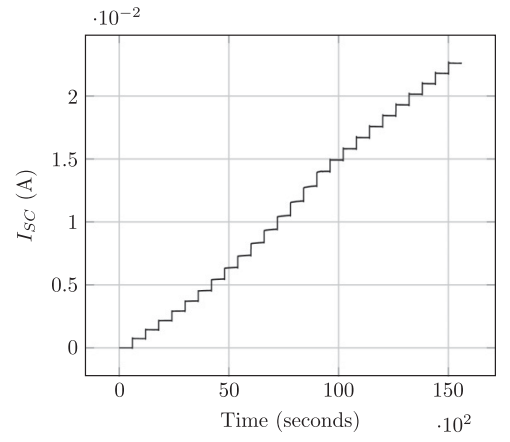

(a) Plot of $I_{S C}$ as PWM is monotonically increased

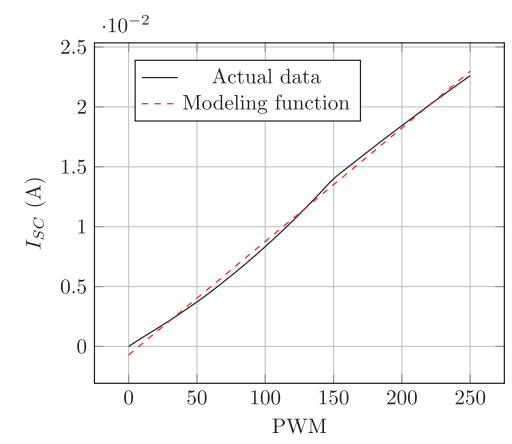

(b) Plot of $I_{S C}$ vs PWM, both from averaged empirical data and a linear regression-produced function

Fig. 5. Plots for characterizing the solar panel - centralized test apparatus configuration.

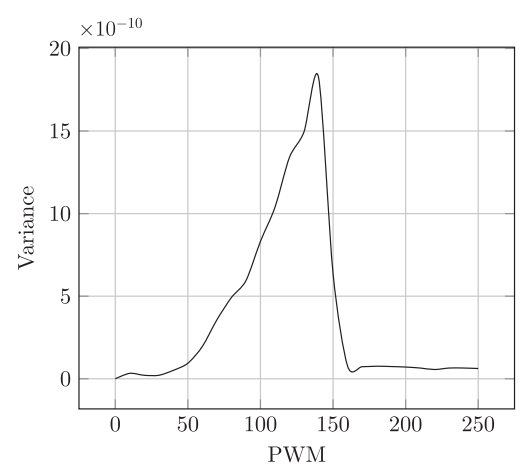

(a) Variance vs PWM

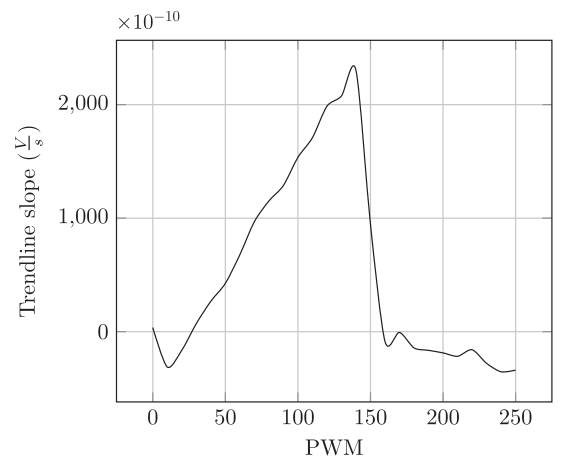

(b) Trendline slope vs PWM

Fig. 6. Stability metrics - centralized test apparatus.

The Arduino allows the user to specify the duty cycle of the PWM signal with 256 values $(0=0 \%$ duty cycle and $255=100 \%$ duty cycle). This corresponds to 256 lighting element settings. To test the stability of the light source (vis-à-vis our recent definition) we test 26 PWM values (multiples of 10, 0 to 250) and measure the resulting solar panel $I_{S C}$. The vertical distance between the solar panel and the LED array is set to $60 \mathrm{~mm}$, and the apparatus is covered during the tests to control the lighting. The test is run continuously, with the PWM value increased in a monotonic manner. The $I_{S C}$ is measured using a Keithley 2401 [Keithley Instruments 2012] sourcemeter connected to a PC running NI Labview [National Instruments 2014]. The Labview program collects a current reading every second, resulting in 600 readings per PWM value tested. The measured $I_{S C}$ values are plotted in Figure 5(a). Figure 5(b) shows the plot of the measured $I_{S C}$ against the PWM values. Figure $5(\mathrm{~b})$ is generated by taking the mean of the 600 current readings taken for each PWM value.

The variance and trendline slope for each set of $I_{S C}$ readings collected are plotted against PWM values in Figure 6(a) and Figure 6(b), respectively. It can be seen in Figure 6 that the light source is not perfectly stable. Nevertheless the light source is still highly stable, exhibiting a maximum variance of $1.825 \times 10^{-9}$ (at PWM value 140) and a maximum trendline slope of $2.3 \times 10^{-7} \frac{\mathrm{V}}{\mathrm{s}}$ (at PWM value 140 ). 


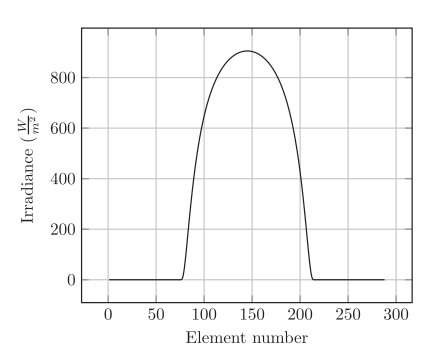

(a) Irradiance

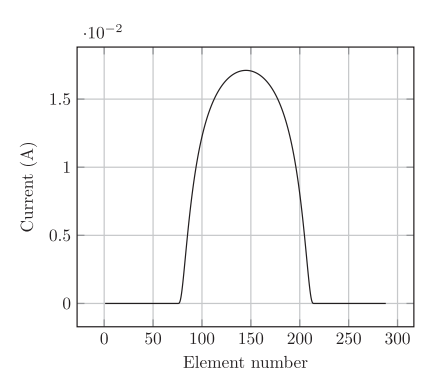

(b) $I_{S C}$

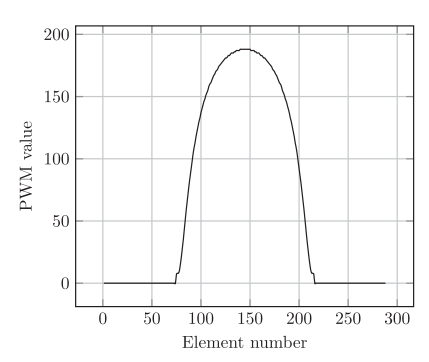

(c) PWM

Fig. 7. Three sequences for September 21, 2014 (autumnal equinox), London.

The extent to which a light source's stability is acceptable is affected by the temporal resolution of irradiance sequence, or the time interval for which a certain irradiance value holds. A larger interval (poorer temporal resolution) gives more opportunities for the $I_{S C}$ to average out and thus tolerate more instability. The experiments are carried out utilizing $5 \mathrm{~min}$ intervals (a $24 \mathrm{~h}$ period is divided into 288 sub-periods). This is long enough, we believe, to accommodate the minimal instability of the light source.

We take this opportunity to underscore a point made earlier in the Introduction about the inadequacy of simple tabletop experiments that do not consult astronomical models. The solar panel we utilize is measured to have a 1 sun $I_{S C}$ of $18.9 \mathrm{~mA}$ (some of its IV curves are plotted in Figure 1). The measurement is done using a calibrated Newport Oriel Sol3A Class AAA $4 \times 4$ inch solar simulator [Oriel Instruments 2012]. As can be seen in Figure 5(b), however, beyond the PWM value of 210, the $I_{S C}$ already exceeds $18.9 \mathrm{~mA}$. This shows how easy it is to place the solar panel in a state it will never be in under natural outdoor conditions.

If the light source is stable, then "day simulation" will simply consist of sequentially placing the light source at the appropriate settings. To do this the $I_{S C}$ sequence will have to be converted to a settings sequence that will then be "played." For our apparatus, the settings sequence is called the PWM sequence.

To convert the $I_{S C}$ sequence to a PWM sequence, we first need to find a function that will approximate the empirical data in Figure 5(b). This can be done in several ways. It is desirable that the function complexity be minimized as much as possible without sacrificing too much accuracy. To this end, we adopt a function generated through linear regression (Equation (8)) as follows:

$$
f(x)=0.000094738695 \times x-0.000707917246 .
$$

Equation (8) is also plotted in Figure 5(b). We can then take the inverse of Equation (8), resulting in a current-to-PWM function. By rounding the output of the currentto-PWM function, an $I_{S C}$ sequence can be converted to a PWM sequence.

As an example of the transformation between the three types of sequences, Figure 7 shows the irradiance sequence, $I_{S C}$ sequence, and PWM sequence for September 21, 2014 , in London, England (coordinates $51.5072^{\circ} \mathrm{N}, 0.1275^{\circ} \mathrm{W}$ ). The astronomical model is evaluated with $5 \mathrm{~min}$ intervals, resulting in a 288 -element sequence for the $24 \mathrm{~h}$ period.

\subsection{Distributed Test System}

There are some tests for which a single test apparatus will not suffice. For example, a single test apparatus cannot test the effect of differing sunlight patterns within a deployment area on network performance. Such tests are important as deployed nodes will likely receive different sunlight patterns because of several factors, including, for 
example, foliage, shadows, or cloud cover. To carry out such tests (which are tests on networks and not simply network nodes), we will need several instances of the test apparatus working together.

Arguably, the apparatus already presented can also be "forced" to test networks. Several instances of the apparatus can be connected using USB cables, and while USB cables are severely limited in length, the power level of the WSN node radios can frequently be adjusted, enabling the implementation of multi-hop networks, even within a single room. Nevertheless, this is an unsatisfactory solution, as it misses many aspects of actual deployments, for instance, the effect of the environment on topology through wave diffraction, diffusion, and refraction.

To this end, we create a version of the test apparatus specifically for testing networks of nodes. Instances of the test apparatus are controlled by a base station, effectively forming a distributed system. Using the original settings sequence as basis, a new sequence can be customized for each test apparatus. To simulate the effect of permanent occlusion such as being under foliage, all the values in the PWM sequence can be attenuated by a certain factor. Temporary occlusion such as temporary cloud cover can be simulated by limiting the attenuation to certain periods. An instance of the test apparatus can be located anywhere within a building, as long as it is close to an electrical socket and within communication range of at least one other test apparatus that will enable it to form a multi-hop path to the base station. Our system can be used in constructing an indoor WSN testbed similar to Motelab [Werner-Allen et al. 2005] and Indriya [Doddavenkatappa et al. 2012] but tailor-made for solar-powered WSNs.

The primary differences between the test apparatus designed for node testing and the test apparatus designed for network testing are in the distribution of the PWM sequence and the generation of the PWM signal. In the test apparatus designed for node testing, PWM signals are generated by an Arduino, which receives the PWM sequence from a PC via a USB cable. In the test apparatus designed for network testing, the PWM signals are generated by a TelosB WSN node, while the PWM sequences are wirelessly received by the same WSN node from the base station. Our base station consists of a PC connected via USB to a TelosB WSN node.

Another difference between the two designs is in the power supply. The centralized version uses a variable-voltage bench power supply, while the distributed version uses a dedicated fixed-voltage power supply unit that is easier to transport. The TelosB WSN node of the distributed test apparatus is currently powered by batteries, although in future design iterations changes will be made so it can also be powered from the fixed-voltage power supply unit.

While the test apparatus designed for node testing uses a box cover for keeping out ambient lighting, the distributed test apparatus uses a dedicated test chamber.

For communicating PWM sequences and synchronization information, the WSN nodes (both at the base station and those at the test apparatus instances) use the Tymo routing protocol/service. Tymo [TinyOS Core Working Group 2008] is the TinyOS [Hill et al. 2000] implementation of the Dynamic Mobile Ad hoc Network (MANET) Ondemand (DYMO) routing protocol [Internet Engineering Task Force: Mobile Ad hoc Networks Working Group 2013]. DYMO is a routing protocol designed by the Internet Engineering Task Force to enable dynamic point-to-point routing between mobile nodes. It was originally designed to run on top of the Internet Protocol.

The schematic of the distributed test apparatus is shown in Figure 8. Figure 9 shows the actual apparatus.

Note that when using the distributed test apparatus, we effectively end up with two co-located WSNs: one for the test bed/distributed test apparatus (composed of TelosBs in our implementation) and the other the network-under-test (NUT). The members of the NUT (labelled "WSN node" Figure 8) need not be TelosBs. The co-location of two 

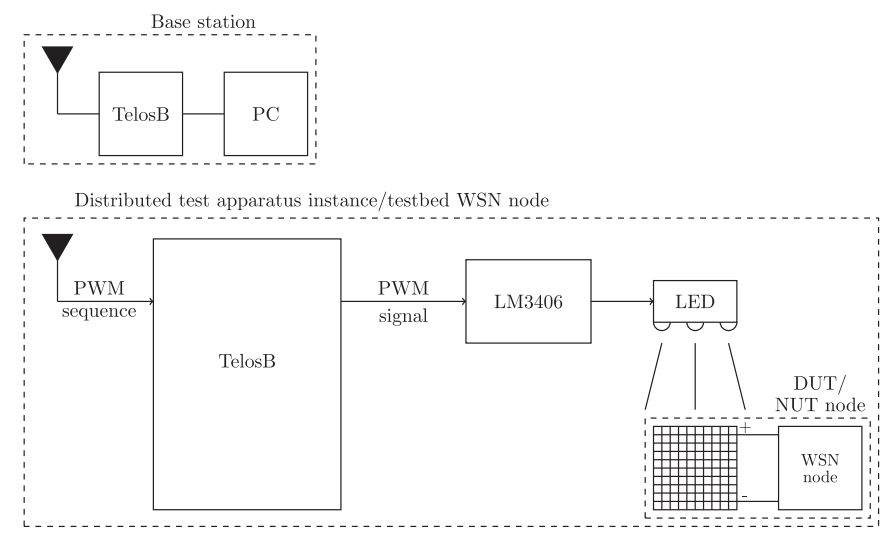

Fig. 8. Design schematic of the implemented distributed test apparatus.

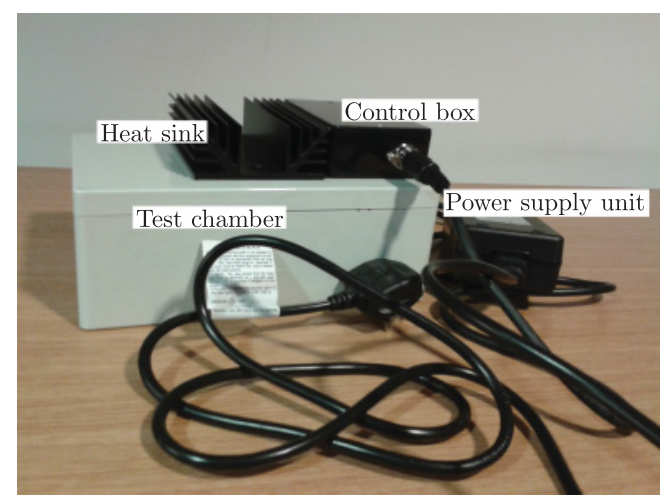

Fig. 9. Distributed test apparatus implementation. TelosB and LM3406 inside control box (beside the heatsink).

WSNs opens up the possibility of interference, which must be minimized. To achieve this, we employ two measures.

First, the communication between the nodes of the testbed WSN is sparse. Such communication only happens at the beginning of the simulation, when the patterns are being distributed, and at the end of the simulation, when the nodes report back to the base station. The post-simulation report enables the base station to ascertain that the simulation was successfully carried out by all nodes. We do not deal with the issue of clock drift or clock synchronization in our current implementation.

Second, we recommend that the two WSNs use different radio channels or frequency sub-bands. The ability to choose channels is a readily available feature even in lowpower radios such as the CC2420 [Texas Instruments 2014], the radio module used in the TelosB. Additional isolation measures were not felt necessary.

To enable the conversion of $I_{S C}$ sequences to PWM sequences suited to the distributed test apparatus, we repeat the experiment performed on the centralized test apparatus. The resulting $I_{S C}$ as the PWM duty cycle is increased is shown in Figure 10(a). It should be noted that Figure 10(a) has differences from Figure 5(a). This is to be expected, since the vertical distance between the solar panel and the LED array differs in the two apparatuses $(50 \mathrm{~mm}$ vs $60 \mathrm{~mm})$. Another difference between the two plots is the range of PWM values. We implement the PWM function of the TelosB using TinyOS' 


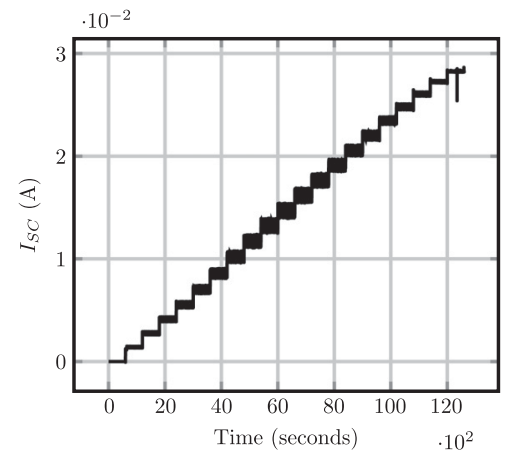

(a) Plot of $I_{S C}$ as PWM is monotonically increased

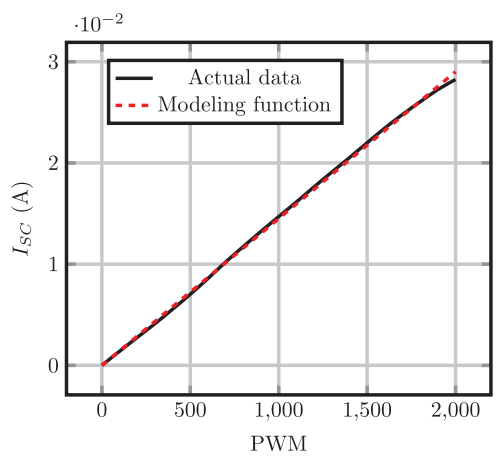

(b) Plot of $I_{S C}$ vs PWM, both from averaged empirical data and linear regressionproduced function

Fig. 10. Plots for characterizing the solar panel: distributed test apparatus.

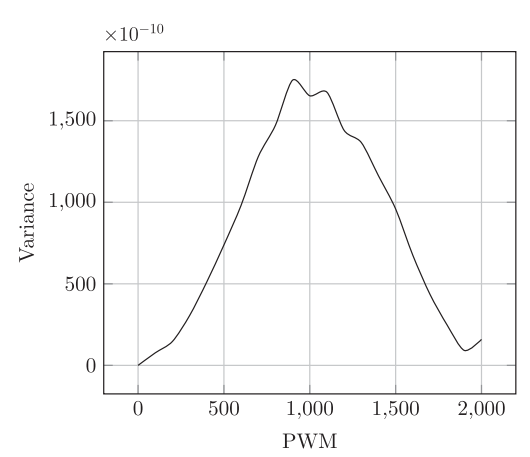

(a) Variance vs PWM

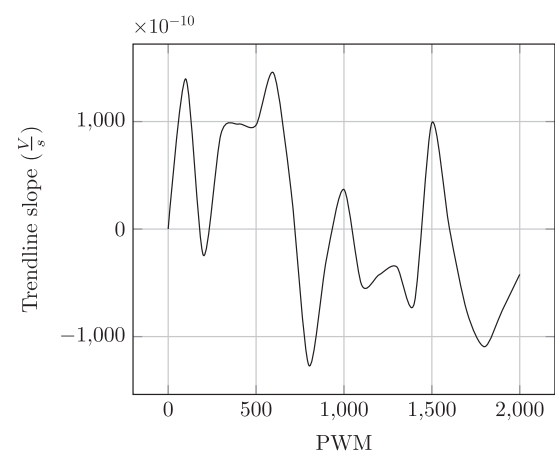

(b) Trendline slope vs PWM

Fig. 11. Stability metrics: distributed test apparatus.

Alarm mechanism, setting the period of the PWM signal to be $2.04 \mathrm{~ms}$ or the same as that of the Arduino PWM signal. However, unlike in the Arduino where the PWM duty cycle can be specified in 256 levels, in our implementation, one can specify the PWM duty cycle in 2,041 levels ( 0 to 2,040 , where $0=0 \%$ duty cycle, and $2,040=100 \%$ duty cycle). We test the PWM values by increments of 100 , from 0 to 2,000 . The results of averaging the $I_{S C}$ values and plotting the averages against the PWM values are shown in Figure 10(b).

We also plot the variance and trendline slope for each set of $I_{S C}$ readings collected in Figure 11(a) and Figure 11(b), respectively. The light source of the distributed test apparatus exhibits a maximum variance of $1.74885 \times 10^{-7}$ (at PWM value 900 ) and a maximum trendline slope of $1.44899 \times 10^{-7} \frac{\mathrm{V}}{\mathrm{s}}$ (at PWM value 600). Similarly to the centralized apparatus, the light source of the distributed apparatus is not perfectly stable, but it is sufficiently stable, we believe, for our application.

We model the empirical data in Figure 10(b) using a function generated through linear regression (Equation (9)), also plotted in Figure 10(b). The inverse of Equation (9) can be used in generating PWM sequences suited for the distributed test apparatus.

$$
f(x)=0.000014511145 \times x-0.000013327296 .
$$




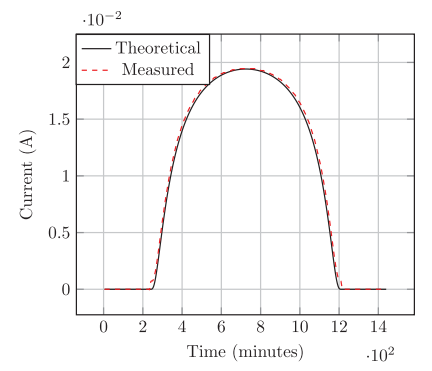

(a) Summer solstice

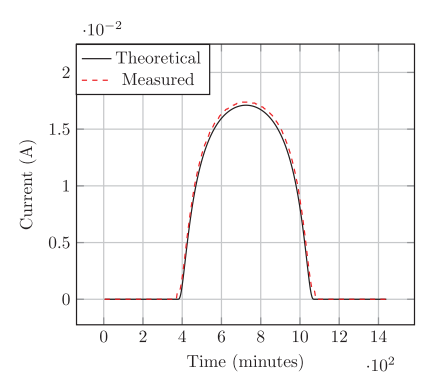

(b) Autumnal equinox

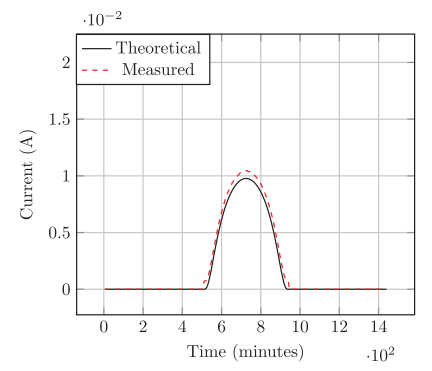

(c) Winter solstice

Fig. 12. Measured and theoretical (target) $I_{S C}$ values: centralized test apparatus.

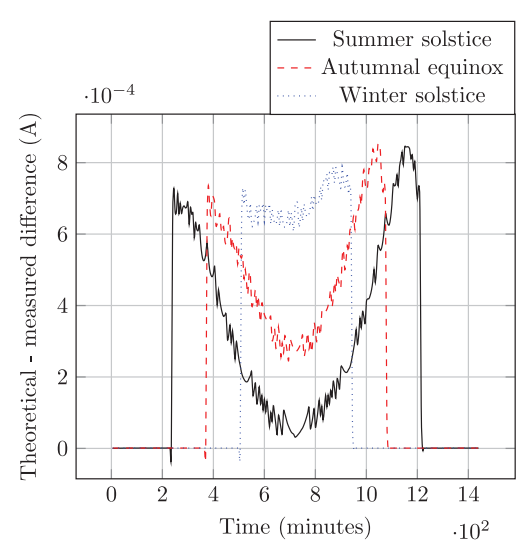

(a) Centralized apparatus

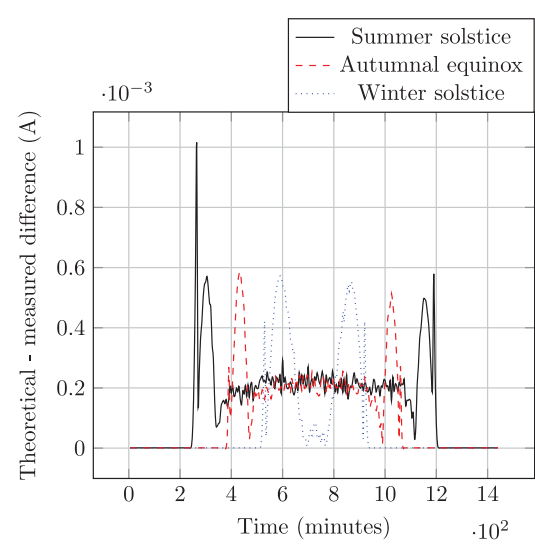

(b) Distributed apparatus

Fig. 13. Difference between theoretical and measured currents.

\section{TEST APPARATUS PERFORMANCE}

The primary purpose of a test apparatus is to induce the solar panel to sequentially produce $I_{S C}$ that correspond to the irradiance values determined by the astronomical model. To test the effectiveness of our test apparatus implementations, we run simulations corresponding to three different dates and compare the resulting $I_{S C}$ values to those in the model-generated sequence. The 3 days simulated are June 21, 2014; September 21, 2014; and December 21, 2014. These dates correspond to the northern hemisphere summer solstice, autumnal equinox, and winter solstice, respectively. The location assumed is the city of London. The astronomical model is evaluated with $5 \mathrm{~min}$ intervals, with the sequence beginning at midnight of the specified day and ending just before the beginning of the next day. The same solar panel ( 1 sun $I_{S C}: 18.9 \mathrm{~mA}$ ) and apparatus setup (DUT-LED array distance: $60 \mathrm{~mm}$ for centralized, $50 \mathrm{~mm}$ for distributed) as those used in Section 4 are used. Consequently, the $I_{S C}$-PWM functions presented in Section 4 are used in generating the PWM sequences. A Keithley 2401 sourcemeter controlled by Labview measures the short circuit current at 1 s intervals. The readings are averaged over $5 \mathrm{~min}$ sets (300 readings in each set). The averaged readings from the centralized test apparatus, along with the target $I_{S C}$ values, are shown in Figure 12. We plot the difference between the theoretical and measured values in Figure 13(a).

In Figure 12 and Figure 13(a), it can be seen that the measured $I_{S C}$ values follow the theoretical $I_{S C}$ values very closely. Between the $5 \mathrm{~min}$ averages, the single maximum difference between measured value and theoretical value is $0.84 \mathrm{~mA}$ for the summer 
solstice (with a mean of $0.245 \mathrm{~mA}, 0.36 \mathrm{~mA}$ if night portion is excluded), $0.85 \mathrm{~mA}$ for the autumnal equinox (with a mean of $0.242 \mathrm{~mA}, 0.49 \mathrm{~mA}$ if night portion is excluded), and $0.789 \mathrm{~mA}$ for the winter solstice (with a mean of $0.2 \mathrm{~mA}, 0.423 \mathrm{~mA}$ if night portion is excluded). For all setups, the maximum deviation occurs in the lowest non-zero PWM values-at sunrise or sunset. Referring to Figure 5(b) (left-side portion), this is to be expected, as this corresponds to the point where the modelling function deviates the most from the empirical data. Another possible source of the deviation is the imperfect stability of the light source.

A prominent feature of Figure 12(c) is the deviation between theoretical and measured $I_{S C}$ in the mid-day section. This can also be explained by the deviation between the empirical data and the modelling function. A closer inspection of Figure 12(a) and Figure 12(b) reveals deviation of roughly the same magnitude in the same $I_{S C}$ range (near 10mA). However, the sequences for the summer solstice and the autumnal equinox spend significantly less time at the said $I_{S C}$ range compared to the winter solstice sequence, so the effect is significantly less overall. The precision therefore with which the test apparatus can replicate an $I_{S C}$ sequence closely depends on the values contained in the sequence. For our specific setup, an $I_{S C}$ sequence with more values that translate to very low non-zero PWM values or are close to $10 \mathrm{~mA}$ will tend to be replicated less precisely than those with less.

Before we discuss how the distributed version performs in replicating the $I_{S C}$ sequences, we note that the distributed apparatus cannot properly generate light for PWM values lower than 50. Instead of dimmed light, what the LED produces at PWM values $0-50$ is light with the maximum level of brightness interspersed with very brief periods of darkness. This is not apparent in Figure 10(a), since the PWM values in Figure 10(a) are tested with increments of 100 beginning at 0 . We attribute the behaviour to how TelosB generates the PWM signal. While the Arduino runs its program "bare metal," TelosB runs TinyOS, which is a simple multi-tasking operating system. The PWM signal is produced by the repeated execution of two tasks. The first task coincides with the beginning of a PWM period and is in charge of pulling the output pin to a value of HIGH. The second task is executed later and pulls the value of the output pin to $L O W$. The interval between the two tasks depends on the duty cycle or PWM value. We use the Alarm mechanism to facilitate the timing of the two tasks. However, at very low PWM values, the interval is so small that the second task is not consistently executed. This leads to periods where the pin value is never pulled down or where the duty cycle generated is effectively $100 \%$ (hence, maximum brightness). This inconsistency is caused by the limitations of the TinyOS Alarm, scheduler, or both. To address this limitation (which would lead to significant errors for the apparatus), we round down all values in the PWM sequence that are lower than 50 to 0 .

The measured $I_{S C}$ values for the distributed test apparatus are shown in Figure 14. We also plot the difference between the theoretical and measured values in Figure 13(b). The distributed test apparatus is accurate in replicating the sequences for all dates tested. Between the 5minaverages, the single maximum difference between measured value and theoretical value is $1.01 \mathrm{~mA}$ for the summer solstice (with a mean of $0.155 \mathrm{~mA}, 0.229 \mathrm{~mA}$ if night portion is excluded), $0.585 \mathrm{~mA}$ for the autumnal equinox (with a mean of $0.1 \mathrm{~mA}, 0.218 \mathrm{~mA}$ if night portion is excluded), and $0.571 \mathrm{~mA}$ for the winter solstice (with a mean of $0.077 \mathrm{~mA}, 0.156 \mathrm{~mA}$ if night portion is excluded). In general, the distributed version outperforms the centralized version when it comes to accuracy. This can be attributed to several factors. The distributed apparatus' lighting mechanism is superior to that of the centralized apparatus in terms of the trendline slope (although it exhibits greater variance). The distributed apparatus' modelling function is also a better fit to the data it models. Finally, PWM values in the distributed test apparatus are represented with the range 0-2,041, compared to 


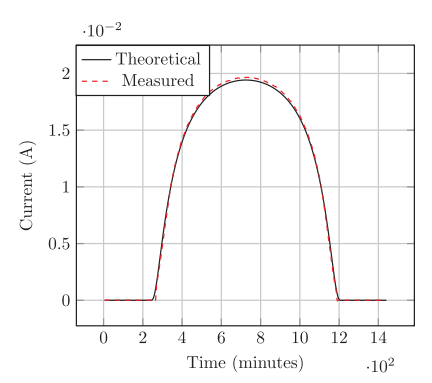

(a) Summer solstice

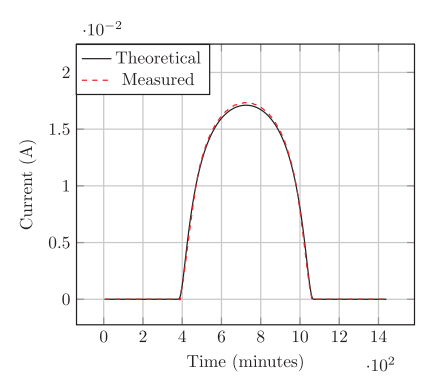

(b) Autumnal equinox

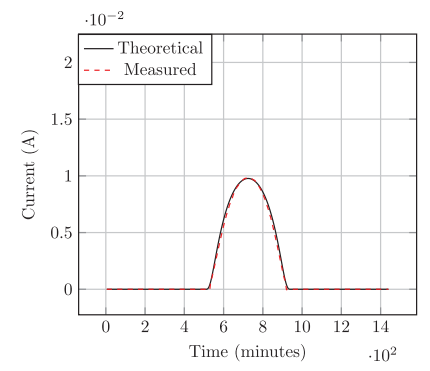

(c) Winter solstice

Fig. 14. Measured and theoretical (target) $I_{S C}$ values: distributed test apparatus.

0-255 in the centralized apparatus. A larger range of numbers translates to greater precision in specifying the PWM signal's duty cycle.

\section{DEMONSTRATION EXPERIMENTS}

To demonstrate the full benefits of the test methodology and the test apparatus, we conduct two experiments, the likes of which are important prior to deploying solar-powered WSNs. We use the centralized version of the test apparatus in the experiments. The energy harvesting component of our setup is a $30 \mathrm{~mm} \times 30 \mathrm{~mm}$ solar panel with $I_{S C}$ of $18.9 \mathrm{~mA}$ (the same as that used in Section 4). The solar panel is connected to a $50 \mathrm{~F}$ supercapacitor formed by placing two 100F Bussmann PowerStor supercapacitors [Cooper Bussmann 2014] in series (the supercapacitor size is varied in the second experiment). The supercapacitor is interfaced to a Direct Current - Direct Current (DC-DC) converter based on the LT1615 [Linear Technology 1998]. The DC-DC converter is configured to accept input voltage levels of $1.5 \mathrm{~V}$ to $3.0 \mathrm{~V}$ and to output a constant $3.3 \mathrm{~V}$, which is used to power the WSN node. The nodes utilized in this study are TelosB nodes running TinyOS. The application running on the node comprises of a simple sleep-send loop, with the send performed through LowPowerListening [TinyOS Core Working Group 2007]. Senders are configured to assume the wakeup interval of the receiver as 1s. All sends are also configured as broadcasts-therefore, at each send, the radio of the node remains active for 1s before returning to sleep. The interval between the sends is a parameter that is varied in the first experiment. The packets from the node are received by a base station that consists of another TelosB node connected via a USB cable to a netbook. The base station keeps track of packets' arrival times and logs the contents of the packets received. The packet has a payload of a single variable that is incremented by the sender node prior to each send. The variable enables us to verify that the node is in continuous operation and that it has not restarted. The physical setup of the test apparatus is the same as that in Section 4. The three simulated days in Section 4 are also used in the two experiments but with some modifications. Instead of starting the sequence at midnight (as is the case in the previous experiments), we start the sequence at sunrise. Each experiment still runs for $24 \mathrm{~h}$; therefore the end of each sequence corresponds very closely to the sunrise of the next day. The majority of the experiments involve the measurement of the supercapacitor voltage. For this, we utilize a Keithley 2401 sourcemeter connected to a PC running NI Labview. The Labview program takes in a voltage reading every minute.

\subsection{Experiment 1: Send Interval}

In the first experiment, we test how the node supercapacitor will evolve for different values of the send interval at different times of the year. We monitor the supercapacitor 


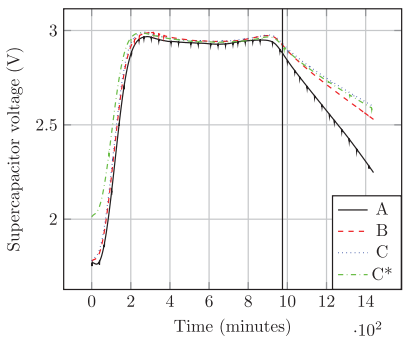

(a) Summer solstice

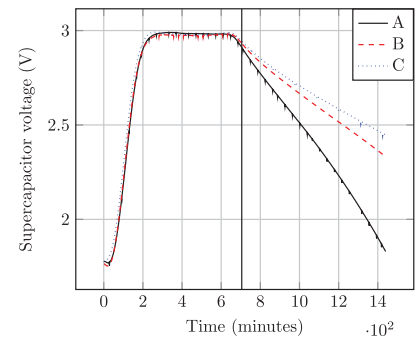

(b) Autumnal equinox

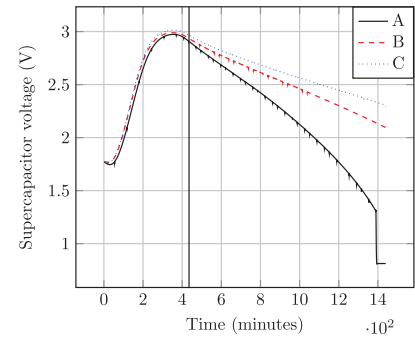

(c) Winter solstice

Fig. 15. Results for the first experiment. Send interval varied: Setup A, 20s send interval; Setup B, 40s send interval; Setup C, 60 s send interval. Initial voltage $2.5 \mathrm{~V}$; $50 \mathrm{~F}$ supercapacitor.

voltage, as it is proportional to the energy stored in the capacitor. This experiment is an example of how algorithm parameters can be tested with the new test methodology. We test three send intervals: $20 \mathrm{~s}, 40 \mathrm{~s}$, and $60 \mathrm{~s}$; we denote the setups with these send intervals as Setups A, B, and C, respectively. The initial supercapacitor voltage is set to $1.767 \mathrm{~V}$. The results for the summer solstice, autumnal equinox, and winter solstice are shown in Figure 15(a), Figure 15(b), and Figure 15(c), respectively. The plots also show the time for the sunset, indicated by a single black vertical line.

From the results, it is apparent that a shorter send interval translates to greater energy consumption: This is especially pronounced during nighttime (right of the vertical line), where we see the voltage for Setup A decreasing faster than then voltage for Setup B, which in turn, decreases faster than the voltage for Setup C. The figures also show that there is a peak level to which the supercapacitor voltages can rise. In Figure 15(a) and Figure 15(b), where there are numerous daylight hours, we see the voltages for all setups remaining at the peak level for extended periods of time. In contrast, in Figure 15(c), the voltage of some setups barely reach the peak before starting to decrease.

In Figure 15(a), it can be seen that for all setups, the final supercapacitor voltage is higher than the initial. This increase in supercapacitor voltage after a $24 \mathrm{~h}$ period indicates an energy surplus-more energy was harvested than was spent during the day. As discussed in Kansal et al. [2007], to ensure energy neutral operation, a daily breakeven or surplus in energy is required: Energy neutral operation is the state of an energy harvesting-powered system where it consumes only an amount of energy equivalent to or less than that what it regularly harvests, thus resulting in potentially perpetual operation. Dynamic power management algorithms can use the increase in voltage level as a cue that the send interval (duty cycle) can be decreased (increased).

The energy surplus for all setups remain during the autumnal equinox (Figure 15(b)). However, the differences between the final and the initial voltage levels decreases. This is to be expected, as the shorter charging periods (the time between sunrise and sunset) lead to less energy being harvested compared to the summer solstice.

The surplus for Setups B and C persists even in the winter solstice (Figure 15(c)), although the differences between the final and initial voltages become smaller than those during the autumnal equinox. As for Setup A, the final voltage level is lower than the initial level, indicating an energy deficit: A deficit happens when more energy is consumed than harvested during the $24 \mathrm{~h}$ period. A deficit implies that the send interval is not sustainable if consecutive days will display the same pattern, since the energy stored in the energy storage device will progressively get lower. Our experiment results suggest that a send interval of 20 s is too short for our system, especially if the goal is to incur a daily energy surplus or breakeven. Dynamic power management algorithms 
can take the calculated deficit as a cue to increase (decrease) the send interval (duty cycle).

Setup A in Figure 15(c) actually exhibits not just a deficit but also a sudden collapse in the supercapacitor voltage that causes the node to stop operating. Continuing the simulation does not cause the node to resume operation, indicating the inability of the design to perform cold booting (the process of booting up via energy harvesting while coming from a very low level of charge).

We note that some deficits are inevitable. Because there is a limit to the energy that a supercapacitor can store, the voltages of some setups with the same power consumption but different initial voltages can converge at the peak level. In the absence of sunlight, the voltages of these setups will decrease in a similar manner, resulting in a common or highly similar final level. We see this in Figure 15(a), with Setups C and C*. Setup $\mathrm{C}^{*}$ has the send interval as Setup $\mathrm{C}$, but with a higher initial voltage of $2.0 \mathrm{~V}$. The range of voltages for which this convergence will happen depends on the length of the day portion with sunlight as well as the power consumption of the node. Long daylight hours give more opportunities for a wider "band" of initial voltage values to eventually converge. Low power consumption makes it easier to reach the peak voltage level, therefore also resulting in a wider band. The final voltage level depends on the power consumption and number of hours without sunlight. A high-enough power consumption and prolonged night could result in a final voltage that is lower than any value in the initial voltage band, resulting in an inevitable deficit. In such a situation, the deficit can only be prevented by decreasing the node power consumption, not by increasing the initial voltage level. Alternatively, it is also possible for the final voltage level to fall within the values in the initial voltage band. In such a situation, those with initial voltage levels higher than the said level will have an inevitable deficit.

\subsection{Experiment 2: Supercapacitor Size}

In the second experiment, we test how the supercapacitor voltage will evolve under different supercapacitor sizes. This is an example of how hardware parameters can be tested with the test methodology. The node utilized has a send interval of $40 \mathrm{~s}$. We test three supercapacitor sizes: $50 \mathrm{~F}, 33.33 \mathrm{~F}$, and $25 \mathrm{~F}$. We denote the setups with these supercapacitors as Setups A, B, and C, respectively. All supercapacitors are formed by placing two or more $100 \mathrm{~F}$ supercapacitors in series.

The energy stored in a capacitor is defined by $E=\frac{1}{2} \times C \times V^{2}$, where $C$ is the size of the capacitor and $V$ is its voltage. For the supercapacitors to have the same initial energy levels, they must have different initial voltages. We choose the values $1.767 \mathrm{~V}$ for Setup A, 2.165V for Setup B, and 2.5V for Setup C. The initial energy is chosen so all supercapacitors have initial voltages higher than $1.5 \mathrm{~V}$ but lower than $3.0 \mathrm{~V}$, the designed operational limits for the DC-DC converter. The results for the summer solstice, autumnal equinox, and winter solstice are documented in Figure 16(a), Figure 16(b), and Figure 16(c), respectively.

It can be seen in the figures that most setups reach the same peak voltage of around 3.0V. During days with long charging periods (daylight hours), the supercapacitor voltages remain for an extended period of time at the peak level. An exception is Setup A during the winter solstice (Figure 16(c)), where the supercapacitor voltage never reaches $3.0 \mathrm{~V}$ before starting to decrease. The presence of a common peak voltage has the consequence that the supercapacitors are able to store different amounts of energy.

To ensure survivability, the energy storage device must be large enough to store sufficient energy for system operation through the hours without sunlight. From our experiments, it is apparent that a $25 \mathrm{~F}$ supercapacitor is insufficient for continuous 


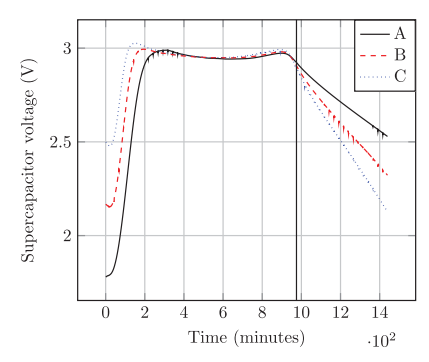

(a) Summer solstice

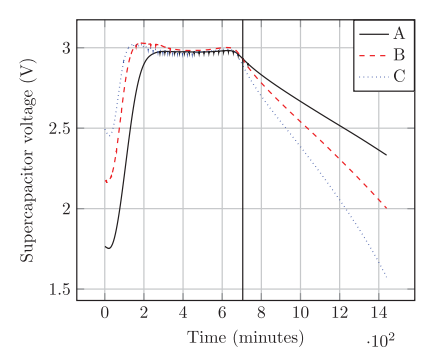

(b) Autumnal equinox

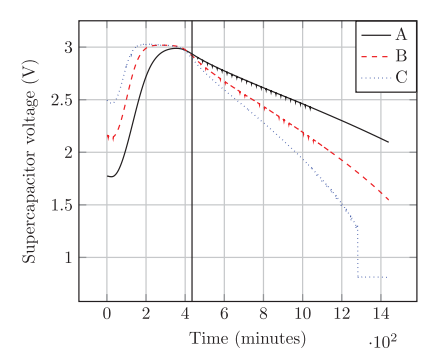

(c) Winter solstice

Fig. 16. Plots for the second experiment. Supercapacitor size varied: Setup A, 50F; Setup B, 33.33F; Setup C, 25F; 40s send interval.

year-long operation: Setup C ends up with a deficit in each day pattern tested, and its supercapacitor voltage collapses during the winter solstice (Figure 16(c)). We note that increasing the initial voltage will not change the situation, since the peak voltage level is already reached by Setup C during the winter solstice. If we assume for simplicity that the peak voltage is attained right before the decrease in voltage due to lack of sunlight, then we calculate that Setups A and C consume 113.53J and 112.01J, respectively, during the winter solstice evening. In comparison, Setups C's peak voltage translates to $114.6 \mathrm{~J}$ of stored energy. Given the very small margin, we can deduce that $25 \mathrm{~F}$ is too small to store the energy required by the system to operate continuously during the winter solstice evening. We also note that at the point of voltage collapse, Setup C theoretically still has 20.48J left in stored energy.

The voltage collapse is a system behaviour that will be missed by simulations that employ simplistic models of the energy storage device. Even relatively complex simulators such as PowerTOSSIM [Shnayder et al. 2004] only shut down nodes when the energy storage device contains 0J-a behaviour clearly not seen in Figure 16(c). Examples of studies where the node is shut down only after completely depleting the energy storage device include Luo et al. [2006] and De et al. [2012].

For a software simulator to capture the emergent system behaviour in Figure 16(c), the simulator must accurately model not just the energy storage device but also the energy harvesting system, which includes the solar panel and the DC-DC converter (software simulation is discussed in greater detail in Section 8.1). The difficulty of finding a correspondence between a real-world system and a simulation model further highlights the need for a methodology that validates and tests designs at the physical implementation level.

Setup B ends up with a surplus during the summer solstice (Figure 16(a)) and deficits during the autumnal equinox (Figure 16(b)) and winter solstice (Figure 16(c)).

Setup A ends up with a surplus in each of the day patterns tested-this indicates that a $50 \mathrm{~F}$ supercapacitor can store enough energy that will sustain the system through any night of the year; whether it will store enough energy is dependent on the energy it has at the beginning of the day and the time of year.

Aside from capacity, operational requirements of other components must also be taken into account when choosing or sizing an energy storage device. For example, it is possible that a large supercapacitor will have the capacity to store enough energy that will power the system through the night but its terminal voltage will fall below the operational limits of the DC-DC converter. This will still result in the system shutting down, as the DC-DC converter is sensitive to the voltage in its input terminals, not directly to the energy stored in the device generating the said voltage. 


\section{LIMITATIONS}

We recognise that the tests employed in this study are approximations of actual daylight patterns. Weather effects clearly mean that the energy that can be harvested at a specific day, time, and place is inherently stochastic. This said, the test methodology that we describe does (i) provide researchers and hardware/software designers with well-defined limits of the energy conditions a node (or network of nodes) may encounter and enables repeatable experiments under these conditions and (ii) provide a framework with which real-world data can be discretised and employed (through Principles 2 and 3 of Section 2.3), should such data exist for a particular region of interest.

Meteorological phenomena can cause the observed irradiation to significantly vary from theoretical values. Irradiance readings are available for some locations. In this study, we deliberately chose London, as the London Air Quality Network [Environmental Research Group, King's College London 2012] makes available past irradiation data for some London boroughs. Other researchers would, therefore, be able to augment this study with additional data should they wish to do so.

Engineers may factor in a test strategy that employs real-world observations before hardware is deployed. In this case, we signal two potential pitfalls with this approach: First, open-source irradiance readings are not available for many locations, and data collection would therefore be necessary. Second, even if such data were available, understanding the predictability of weather patterns is difficult (as medium- to long-term weather forecasting shows us), and some mathematical modelling would be necessary. It is for these reasons, and the aim for a generic apparatus and methodology, that our work takes the approach that it does.

This test methodology should not be used to compare different solar panels. It can, however, be used for testing (and sizing) different components "downstream" of the solar panel such as the DC-DC converter, the energy storage device, and the microcontroller. For testing different solar panels, a solar simulator must be used. Another advantage of using a solar simulator (assuming that its irradiance can be continuously and automatically varied) is that it will remove the need to derive the $I_{S C}$ vs PWM function, thus simplifying the test methodology.

A further limitation of the current test methodology and test apparatus is that they do not take into account the solar panel's temperature. The temperature of a solar panel can have an effect on its performance. A test apparatus that takes into account and controls the temperature of the solar panel can be constructed by incorporating features of TempLab [Boano et al. 2014]. TempLab is an extension for WSN testbeds that allows the control of node temperatures by using infra-red light bulbs and Peltier cooling modules.

\section{RELATED WORK}

To the best of our knowledge, there are no other studies concerning the indoor testing of solar-powered WSNs without the use of solar simulators. We therefore focus on the test methodologies and power models used in studies concerning solar-powered or energyharvesting WSNs. We divide such test methodologies into two categories: those that utilize software-based simulations and models (Section 8.1) and those that are used with real-world systems (Section 8.2).

\subsection{Software-Based Simulations and Models}

8.1.1. Numerical Simulations. Most studies on power management algorithms that rely on scheduling tasks (such as Moser et al. [2006] and Chetto and Zhang [2010]) test their algorithms using numerical simulations with very simple power models. The 
simulators utilized in these studies are mostly custom-made and implemented in $\mathrm{C}$ or MatLab. The utilization of custom simulators makes experimental comparison and verification difficult. A more significant problem with the test methodology employed in these studies is their sheer simplicity. Hardware non-idealities and the environment are rarely taken into account, therefore, significant work and verification using a methodology suitable for actual devices is required before such algorithms are feasible for actual systems or devices.

8.1.2. Discrete Event Simulator Extensions. An additional approach to simulating the power consumption of energy-harvesting nodes utilizes extensions to existing simulation frameworks. Notable examples of this approach are SensorSim [Park et al. 2000] and PowerTOSSIM [Shnayder et al. 2004]. PowerTOSSIM [Shnayder et al. 2004] is an extension to TOSSIM [Levis et al. 2003] which enables the simulation and measurement of the energy consumption of TinyOS applications. When running a simulation, PowerTOSSIM analyses the instructions in the program and, depending on the components utilized by the instruction (and the states the components are in), computes the node power consumption.

The accuracy of the power values in these simulators is usually improved by using values derived from hardware profiling. In PowerTOSSIM, for example, a hardware power consumption model is built using microbenchmarks that exercise each hardware component independently. Power consumption readings are taken while microbenchmarks are running in the node. An example of a tool for power metering of wireless sensor nodes is Quanto [Fonseca et al. 2008].

The main drawback of this approach is the difficulty of carrying out hardware profiling, and, in the case of TOSSIM and PowerTOSSIM, creating an accurate instructioncomponent model. TinyOS supports several WSN node platforms (Mica [Hill and Culler 2002], Mica2, MicaZ, and TelosB, to mention but a few), but TOSSIM and PowerTOSSIM currently only support the MicaZ platform. An additional drawback is that these simulator extensions only consider the node itself and not the components usually added to node platforms to enable them to harvest energy. PowerTOSSIM, for instance, does not take into account DC-DC converter efficiency, leakage in the energy storage device, or the environment (sunlight patterns).

\subsection{Test Methodologies for Actual Physical Systems}

Most systems destined for actual deployments are not tested indoors prior to deployment. Instead, the energy consumption of the node (in joules per operation, for instance) and the energy that can be harvested from the environment are estimated, and the parameters (such as the application duty cycle) are subsequently set. This approach has obvious drawbacks: The performance (and survivability) of the system will depend on the accuracy of the estimates, and such estimates are difficult to acquire with high precision because of factors such as hardware non-idealities and the inherently stochastic nature of the energy that can be harvested from the environment. To compensate, conservative parameters are often used, but this leads to loss of performance and will still not be a guarantee of system survivability.

One such study that employed the "estimate-and-deploy" methodology is described in Taneja et al. [2008]. They describe an approach used in designing the energy harvesting subsystem of sensor nodes that were used in studying hydrological cycles in forest watersheds. The computations underlying the choice and sizing of components are explained, as well as the rationale for key design decisions. The application that was run on the node is not adaptive: It operated at the same duty cycle, regardless of the environmental conditions. While best effort was made in estimating the parameters, nodes still shut down during the deployment. 
The duty cycling scheme presented in Lee et al. [2010] was tested on actual hardware (TI eZ430-RF2500-SEH [Texas Instruments 2009]), with the solar panels being illuminated by a desktop lamp. However, the brightness of the lamp was not calibrated in any way, nor was it cycled or modulated to reflect an actual day pattern. Given the lack of calibration, it is very much possible that the energy harvesting system was induced to produce energy higher than it will under actual outdoor conditions. As we highlighted in Section 4, this is a problem that will lead to poorly developed systems.

A significant departure from the methods used in most other work, Jeong et al. [2008] performed experiments (comparison between different energy harvesting WSN node designs) outdoors under natural sunlight: The experiments were done during "sunny days in mid-October" to minimize weather effects. The experiments performed, while valid, are impossible to replicate and do not capture seasonal effects.

A drawback with the reliance on actual deployments for testing is that no twoday patterns are exactly the same: Because of this lack of repeatability, parameters, algorithms, and designs are difficult to objectively compare. Even if the variability between day patterns is ignored, as in Jeong et al. [2008], relying on actual deployments means being restricted by one's location and the current season. For example, to test how the system will perform during winter, the study would have to be performed in winter. A system destined for deployment in the Arctic will have to be tested, at all stages, in the Arctic.

Our test methodology, in comparison, enables the repeatable testing of parameters, algorithms, and hardware designs. The studies that result are not limited by the current season or the location where the study is being conducted. How a node (or even network of nodes) will fare in sub-Saharan Africa during winter can be studied in a controlled laboratory experiment.

\subsection{Summary and Comparison}

Previous research cited above has been developed to support specific test scenarios and/or different stages of the WSN development cycle. Early-stage investigation of power management algorithms will typically be implemented using custom simulators and associated power models; these may themselves rely on many assumptions but allow the design process to be steered toward more effective solutions. Later-stage hardware designs, or the tuning of software that is already in deployment, would hope to replace earlier assumptions with real-world data. As we state in Section 8.2, experimental repeatability is a serious concern in actual deployments and impacts this later-stage design engineering. Our methodology, in contrast to previous research, provides the benefits of a hardware-based approach (real-world properties of actual hardware), together with some of the flexibility of software-based simulation (the lowcost solar apparatus can be repeatedly configured alongside the energy-harvesting algorithms), offering a unique approach to this problem.

\section{CONCLUSION AND FUTURE WORK}

We present an indoor test methodology for solar-powered WSNs. The methodology is based on astronomical models and PV cell design principles, and it enables repeatable experiments without the need for expensive solar simulators. We detail the design of a generic test apparatus that can be used in implementing the test methodology. We also detail the design of our own implemented test apparatus, which has two variants: centralized and distributed. The centralized version can be used in experiments involving isolated solar-powered WSN nodes. The distributed version can be used in testing networks of such nodes. Our implemented designs differ from the generic design in that they do not rely on a feedback loop as a control mechanism, relying instead on the stability of the light source and a modelling function specifying the relationship 
between the $I_{S C}$ and the lighting element setting. The omission of the feedback loop significantly simplifies the apparatus design.

Our experiments show that the implemented test apparatuses can replicate target $I_{S C}$ sequences accurately, although the accuracy varies depending on the specific $I_{S C}$ value involved. The variations in accuracy between different $I_{S C}$ values stem from the light source's imperfect stability and inaccuracies in the modelling function.

We also perform a series of experiments demonstrating how the test methodology can be used in deriving software and hardware parameters for a WSN node. The results of our experiments largely confirm well-known power management principles. We note, however, that prior to this work, deriving such parameters through repeatable experimentation has been impossible (at least not without a solar simulator).

The study we present is primarily an enabling study and can be applied in many other research areas involving actually implemented solar-powered WSNs or WSN nodes. For example, it can be used in designing and studying dynamic power management algorithms or testing the ability of hardware designs to perform cold booting.

Future research can proceed in several directions. Hardware improvements can be made: As embedded computing platforms become cheaper, smaller, and more powerful, we can integrate greater capability into the test apparatus. For example, measurement of the short-circuit current might, in future variants, be integrated with the apparatus itself (removing the need to derive $I_{S C}$ vs PWM beforehand). Similarly, with smaller and more power-efficient WiFi (802.11) transceiver modules, we also envisage the distributed test apparatus being run over Transmission Control Protocol/Internet Protocol (TCP/IP), with supporting control over the Internet. Alternatives to the astronomical model presented, such as that found in Poggi et al. [2000], might also be tested to see whether they are more representative of external conditions. We envisage customised models being created for specific locations and times using historical meteorological data. Any model can theoretically be used, providing their outputs can be converted to an $I_{S C}$ equivalent. Finally, our approach of subjecting nodes to controlled environmental conditions to simulate actual energy harvesting can be applied to energy harvesting WSNs (and WSN nodes) powered by sources other than solar energy, including wind, vibration, and ambient radio frequency energy.

\section{REFERENCES}

Karl Astrom and Tore Hagglund. 1995. PID Controllers: Theory, Design and Tuning (2nd ed.). International Society of Automation (ISA), North Carolina, Chapter 3.

Carlo Alberto Boano, Marco Zúñiga, James Brown, Utz Roedig, Chamath Keppitiyagama, and Kay Römer. 2014. TempLab: A testbed infrastructure to study the impact of temperature on wireless sensor networks. In Proceedings of the 13th International Symposium on Information Processing in Sensor Networks (IPSN'14). IEEE Press, 95-106.

Maryline Chetto and Hui Zhang. 2010. Performance evaluation of real-time scheduling heuristics for energy harvesting systems. In Green Computing and Communications (GreenCom), 2010 IEEE/ACM Int'l Conference on Int'l Conference on Cyber, Physical and Social Computing (CPSCom). ACM, New York, NY, 398-403. DOI : http://dx.doi.org/10.1109/GreenCom-CPSCom.2010.16

Debraj De, Wen-Zhan Song, Shaojie Tang, and Diane Cook. 2012. EAR: An energy and activity-aware routing protocol for wireless sensor networks in smart environments. Comput. J. (2012). DOI: http:// dx.doi.org/10.1093/comjnl/bxs039

Manjunath Doddavenkatappa, MunChoon Chan, and A. L. Ananda. 2012. Indriya: A low-cost, 3d wireless sensor network testbed. In Testbeds and Research Infrastructure. Development of Networks and Communities, Thanasis Korakis, Hongbin Li, Phuoc Tran-Gia, and Hong-Shik Park (Eds.). Lecture Notes of the Institute for Computer Sciences, Social Informatics and Telecommunications Engineering, Vol. 90. Springer, Berlin, New York, NY, 302-316. DOI : http://dx.doi.org/10.1007/978-3-642-29273-6_23

Rodrigo Fonseca, Prabal Dutta, Philip Levis, and Ion Stoica. 2008. Quanto: Tracking energy in networked embedded systems. In Proceedings of the 8th USENIX Conference on Operating Systems Design and Implementation (OSDI'08). USENIX Association, Berkeley, CA, 323-338. 
Jason Hill, Robert Szewczyk, Alec Woo, Seth Hollar, David Culler, and Kristofer Pister. 2000. System architecture directions for networked sensors. SIGPLAN Not. 35, 11 (Nov. 2000), 93-104. DOI:http:// dx.doi.org/10.1145/356989.356998

Jason L. Hill and David E. Culler. 2002. Mica: A wireless platform for deeply embedded networks. IEEE Micro 22, 6 (Nov. 2002), 12-24. DOI : http://dx.doi.org/10.1109/MM.2002.1134340

Internet Engineering Task Force: Mobile Ad hoc Networks Working Group. 2013. Dynamic MANET Ondemand (AODVv2) Routing (draft-ietf-manet-dymo-26). Retrieved June 12, 2015 from http://tools.ietf. org/html/draft-ietf-manet-dymo-26.

Jaein Jeong, Xiaofan Jiang, and David Culler. 2008. Design and analysis of micro-solar power systems for wireless sensor networks. In Proceedings of the 5th International Conference on Networked Sensing Systems (INSS'08). IEEE, New York, NY, 181-188. DOI : http://dx.doi.org/10.1109/INSS.2008.4610922

Aman Kansal, Jason Hsu, Sadaf Zahedi, and Mani B. Srivastava. 2007. Power management in energy harvesting sensor networks. ACM Trans. Embed. Comput. Syst. 6, 4, Article 32 (Sept. 2007). DOI : http://dx. doi.org/10.1145/1274858.1274870

Pius W. Q. Lee, Mingding Han, Hwee-Pink Tan, and Alvin Valera. 2010. An empirical study of harvestingaware duty cycling in environmentally-powered wireless sensor networks. In 2010 IEEE International Conference on Communication Systems (ICCS). IEEE, New York, NY, 306-310. DOI:http://dx. doi.org/10.1109/ICCS.2010.5686442

Philip Levis, Nelson Lee, Matt Welsh, and David Culler. 2003. TOSSIM: Accurate and scalable simulation of entire TinyOS applications. In Proceedings of the 1st International Conference on Embedded Networked Sensor Systems (SenSys'03). ACM, New York, NY, 126-137. DOI : http://dx.doi.org/10.1145/958491.958506

Jun Luo, Jacques Panchard, MichałPiórkowski, Matthias Grossglauser, and Jean-Pierre Hubaux. 2006. MobiRoute: Routing towards a mobile sink for improving lifetime in sensor networks. In Proceedings of the Second IEEE International Conference on Distributed Computing in Sensor Systems (DCOSS'06). Springer-Verlag, Berlin, 480-497. DOI : http://dx.doi.org/10.1007/11776178_29

Tomas Markvart (Ed.). 2000. Solar Electricity (2nd ed.). John Wiley \& Sons, Chichester, England, Chapter 2.

Arduino. 2013. Arduino. (Nov. 2013). Retrieved June 12, 2015 from http://www.arduino.cc/.

Cooper Bussmann. 2014. Cooper Bussmann PowerStor supercapacitors: HV Series. Retrieved June 12, 2015 from http://www.cooperindustries.com/content/dam/public/bussmann/Electronics/Resources/productdatasheets/Bus_Elx_DS_4376_HV_Series.pdf.

Environmental Research Group, King's College London. 2012. London Air Quality Network. Retrieved September 4, 2015 from http://www.londonair.org.uk/.

Keithley Instruments. 2012. 2401 Low Voltage Sourcemeter Instrument. (Jan. 2012). Retrieved June 12, 2015 from http://www.keithley.com/data?asset=55849.

Linear Technology. 1998. LT1615/LT1615-1: Micropower Step-Up DC/DC Converters in ThinSOT. Retrieved June 12, 2015 from http://cds.linear.com/docs/en/datasheet/16151fas.pdf.

Linear Technology. 2013. LT6105: Precision, Extended Input Range Current Sense Amplifier. Retrieved June 12, 2015 from http://cds.linear.com/docs/en/datasheet/6105fa.pdf.

Lumileds. 2014. LUXEON K: Plug and play matrix solution with precise flux, $V_{f}$ and color. Retrieved June 12, 2015 from http://www.lumileds.com/uploads/366/DS102-pdf.

National Instruments. 2014. LabVIEW System Design Software. Retrieved June 12, 2015 from http://www. ni.com/labview/.

Oriel Instruments. 2012. Oriel Sol3A Class AAA Solar Simulators. Retrieved June 12, 2015 from http:// assets.newport.com/webDocuments-EN/images/DS-12082_Sol3_Solar_Sim.pdf.

Texas Instruments. 2009. eZ430-RF2500-SEH: Solar energy harvesting development kit. Retrieved June 12, 2015 from http://www.ti.com/lit/ml/sprt506/sprt506.pdf.

Texas Instruments. 2013. LM3406: 1.5-A, Constant Current, Buck Regulator for Driving High Power LEDs. Retrieved June 12, 2015 from http://www.ti.com/lit/ds/symlink/lm3406.pdf.

Texas Instruments. 2014. CC2420: 2.4 GHz IEEE 802.15.4/ZigBee-ready RF Transceiver Datasheet. Retrieved from http://www.ti.com/lit/ds/symlink/cc2420.pdf.

TinyOS Core Working Group. 2007. TinyOS TEP 105: Low Power Listening. Retrieved June 12, 2015 from http://www.tinyos.net/tinyos-2.x/doc/html/tep105.html.

TinyOS Core Working Group. 2008. Tymo. Retrieved June 12, 2015 from http://tinyos.stanford.edu/tinyoswiki/index.php/Tymo.

Aden B. Meinel and Marjorie P. Meinel. 1976. Applied Solar Energy: An Introduction (1st ed.). AddisonWesley, MA, USA. 
Roger A. Messenger and Jerry Ventre. 2010. Photovoltaic Systems Engineering (2nd ed.). CRC Press, Boca Raton, FL, Chapter 2.

Clemens Moser, Davide Brunelli, Lothar Thiele, and Luca Benini. 2006. Real-time scheduling with regenerative energy. In Real-Time Systems, 2006. 18th Euromicro Conference on. IEEE, New York, NY, 260-270. DOI : http://dx.doi.org/10.1109/ECRTS.2006.23

Sung Park, Andreas Savvides, and Mani B. Srivastava. 2000. SensorSim: A simulation framework for sensor networks. In Proceedings of the 3rd ACM International Workshop on Modeling, Analysis and Simulation of Wireless and Mobile Systems (MSWIM'00). ACM, New York, NY, 104-111. DOI:http://dx.doi. org/10.1145/346855.346870

Philippe Poggi, Gilles Notton, Marc Muselli, and Alain Louche. 2000. Stochastic study of hourly total solar radiation in Corsica using a Markov model. Int. J. Climatol. 20, 14 (2000), 1843-1860. DOI: http:// dx.doi.org/10.1002/1097-0088(20001130)20:14<1843::AID-JOC561>3.0.CO;2-O

Joseph Polastre, Robert Szewczyk, and David Culler. 2005. Telos: Enabling ultra-low power wireless research. In Proceedings of the 4th International Symposium on Information Processing in Sensor Networks (IPSN'05). IEEE Press, Article 48.

Victor Shnayder, Mark Hempstead, Bor-rong Chen, Geoff Werner Allen, and Matt Welsh. 2004. Simulating the Power Consumption of Large-scale Sensor Network Applications. In Proceedings of the 2nd International Conference on Embedded Networked Sensor Systems (SenSys'04). ACM, New York, NY, 188-200. DOI : http://dx.doi.org/10.1145/1031495.1031518

Jay Taneja, Jaein Jeong, and David Culler. 2008. Design, modeling, and capacity planning for micro-solar power sensor networks. In Information Processing in Sensor Networks, 2008. IPSN'08. International Conference on. ACM, New York, NY, 407-418. DOI :http://dx.doi.org/10.1109/IPSN.2008.67

Geoffrey Werner-Allen, Patrick Swieskowski, and Matt Welsh. 2005. MoteLab: A wireless sensor network testbed. In Proceedings of the 4th International Symposium on Information Processing in Sensor Networks (IPSN'05). IEEE Press..

Received June 2015; revised September 2016; accepted September 2016 